\title{
Evolución histórica del concepto y criterios actuales para el diagnóstico de demencia.
}

Historical evolution of the concept and new diagnostic criteria for Dementia.

\author{
Nilton Custodio ${ }^{1,2, a, b, c}$, Rosa Montesinos ${ }^{1,3, d}$, Jorge O. Alarcón ${ }^{4,5, e}$
}

\section{RESUMEN}

A mediados del siglo XIX se empezó a enfocar el concepto de Demencia, como una entidad irreversible asociada a la edad, Esquirol fue uno de los primeros en plantear una aproximación descriptiva del cuadro clínico, y Bayle el primero en proponer Demencia como una enfermedad atribuible a lesiones en el sistema nervioso central. Fue alrededor del año 1900, que Alois Alzheimer precisó las lesiones patológicas de dos entidades clínicas distintas, la primera descrita por él mismo, y la segunda por Arnold Pick (conocidas hoy como enfermedad de Alzheimer y demencia frontotemporal respectivamente). Conforme progresaron las técnicas de imágenes cerebrales y estudios de líquido cefalo-raquídeo fueron mejorando los criterios de diagnóstico para los diversos tipos de demencia, con un enfoque clínico-biológico. Las recomendaciones más recientes, sin embargo proponen un enfoque exclusivamente biológico de la enfermedad de Alzheimer.

PALABRAS CLAVE: Demencia, diagnóstico, enfermedad de Alzheimer, historia. (Fuente: DeCS BIREME).

\section{SUMMARY}

By the middle of the $19^{\text {th }}$ Century the concept of Dementia, as an irreversible entity associated with the aging process, came into focus thanks to the work of Esquirol, who was one of the first to propose a descriptive approach to the clinical picture, and Bayle, who was the first to suggest that Dementia was an illness attributable to injuries of the Central Nervous System. Around 1900, Alois Alzheimer described the pathology of the injuries of two distinct entities, the first described by him, and the second by Arnold Pick (known today as Alzheimer's Disease and Frontotemporal Dementia respectively). The advances in brain-imaging techniques and studies of cerebrospinal fluid led to improvements of the clinical diagnostic criteria for the diverse types of dementia, with a clinicalbiological focus. Nevertheless, the most recent recommendations suggest Alzheimer's disease to be approached from a purely biological focus.

KEYWORDS: Dementia, diagnosis, Alzheimer disease, history. (Source: MeSH NLM).

1 Unidad de Diagnóstico de Deterioro Cognitivo y Prevención de Demencia, Instituto Peruano de Neurociencias. Lima, Perú.

2 Servicio de Neurología, Instituto Peruano de Neurociencias. Lima, Perú.

3 Servicio de Medicina de Rehabilitación, Instituto Peruano de Neurociencias. Lima, Perú.

4 Centro de Investigaciones Tecnológicas, Biomédicas y Medioambientales. Lima, Perú.

5 Departamento de Medicina Preventiva y Salud Pública, Universidad Nacional Mayor de San Marcos. Lima, Perú.

a Médico Neurólogo; ${ }^{\mathrm{b}}$ Magister en Neurociencias; ${ }^{\mathrm{a}}$ Magister en Medicina; ${ }^{\mathrm{d}}$ Médica Rehabilitadora; ${ }^{\mathrm{e}}$ Magister en Salud Pública 


\section{INTRODUCCIÓN}

El Manual diagnóstico y estadístico de las enfermedades mentales (DSM por sus siglas del inglés Diagnostic and statistical manual of mental disorders) de la American Psychiatric Association (APA) en su quinta edición (DSM 5)-versión traducida al español, publicado el 2014, define demencia como un síndrome que incluye la pérdida de funciones cognitivas con compromiso de la funcionalidad, es decir pérdida de las actividades de vida diaria para el funcionamiento laboral, social y familiar (1), sin mencionar los síntomas psicológicos y conductuales, que en algunos casos pueden ser las primeras manifestaciones (como apatía/desinhibición en demencia fronto-temporal o depresión como pródromo de la enfermedad de Alzheimer) (2), cuya frecuencia se incrementa (agitación/agresividad, ansiedad, alucinaciones visuales, delusiones paranoides, entre otros) conforme evoluciona en severidad el síndrome de demencia (3). A pesar de esta omisión, esta definición nos permite reconocer diferentes subtipos de demencia basado en el perfil del deterioro cognitivo, funcional y psicológicoconductual; en el comportamiento estructural (tomografía o resonancia magnética cerebral) y funcional (resonancia magnética cerebral funcional , tomografía por emisión de fotón único o tomografía por emisión de positrones) de las neuroimágenes y en la evaluación de ciertos biomarcadores (4); también nos permite armonizar los criterios para el diagnóstico de demencia y por ende establecer estrategias de prevención que impacten en las políticas públicas, particularmente en países en vías de desarrollo $(5,6)$. Sin embargo, de acuerdo a la evolución del conocimiento y precisando las características clínicas no ha sido posible definir los diferentes tipos de demencia, por lo que los investigadores empezaron a plantearlas como entidades clínico-biológicas, particularmente las demencias degenerativas como la enfermedad de Alzheimer (EA) donde los síntomas clínicos se relacionaban a ciertas alteraciones de proteínas cerebrales (amiloide y tau) evidenciado a través de neuro-imágenes o en líquido cefalorraquídeo. Recientemente, se ha planteado definir a la EA como un constructo biológico (7), donde los síntomas clínicos pasan a un segundo plano, mientras que los biomarcadores son la base del diagnóstico, en el cual el depósito de proteínas anormales la definen como una enfermedad neuro-degenerativa única entre diferentes desórdenes que pueden desarrollar demencia. De esta manera, se podría tener una caracterización más precisa, un mejor conocimiento de la secuencia de eventos que conducen al deterioro cognitivo, así como de la etiología multi-factorial de la demencia; pero sobre todo va a permitir seleccionar con mayor precisión a los pacientes que ingresarán a ensayos clínicos con drogas específicas según la vía etiopatogénica del proceso de la enfermedad, es decir medicación anti-amiloide o anti-tau. Aunque se precisa que estos criterios solo son aplicables para investigación y no para la práctica clínica diaria, queda claro que la precisión del concepto de demencia ha sufrido diversas variaciones desde sus orígenes, por lo que la manera más didáctica de entenderlo es precisando la diferencia entre la evolución del "termino de demencia" y la evolución del "concepto de demencia". Con la finalidad de conocer los aspectos históricos del concepto de demencia y de los criterios del diagnóstico de demencia, revisamos en detalle las diversas publicaciones sobre la historia del diagnóstico de demencia, el deterioro cognitivo leve, la enfermedad de Alzheimer, el trastorno cognitivo vascular, la demencia vascular, la demencia fronto-temporal, la demencia con cuerpos de Lewy y la demencia mixta. Para ello, revisamos estudios publicados en las siguientes bases de datos: Medline, Biomed Central, Embase, Scopus, Scirus, PsychInFO, LILACS y Scielo. En forma independiente, los autores buscamos los siguientes términos: (Dementia) AND (Alzheimer's disease) AND (Cognitive impairment) AND ("history" OR "diagnosis" OR "classification" OR "nosology" OR "DSM" OR "NIA-AA" OR "biomarkers"). Incluimos publicaciones en idioma español, portugués e inglés. El periodo de búsqueda incluyó artículos originales y de revisión publicados desde 1980 hasta 31 de mayo de 2018. Después de leer resúmenes y textos completos, nuestra búsqueda identificó 60 publicaciones sobre historia del diagnóstico de demencia y términos relacionados.

\section{Historia del concepto de demencia}

Etimológicamente "demencia" está compuesta por el prefijo de (ausencia), mente (mente) y el sufijo ia (condición o estado), por lo que se podría asumir que demencia es aquella "condición en la cual se pierde la mente" (8), definición que en la actualidad se aplicaría sólo a estadios avanzados de demencia. Para tener un conocimiento más preciso de la evolución del concepto de demencia, es pertinente separar la "historia del término demencia" de la "historia del concepto de demencia como enfermedad" $(9,10)$. Durante siglos, el término demencia se usó con un sentido diferente al del concepto actual de demencia. Por otro lado, la evolución del concepto de deterioro cognitivo también se inició en la misma época y lugar. A partir del siglo 
XVII, la descripción de la semiología de los trastornos cognitivos se hizo más precisa, se separaron los trastornos congénitos de los retardos mentales y de los trastornos cognitivos adquiridos, se distinguieron los trastornos cognitivos de otras enfermedades mentales y se reconoció que el deterioro cognitivo se explica por un disfuncionamiento del sistema nervioso central (9-12). Recién en el siglo XIX, el "término" demencia y el "concepto" de demencia confluyen, al comenzar a usarse la palabra demencia en el sentido que tiene en la literatura médica actual.

La primera alusión escrita e imprecisa sobre el término demencia se encuentra en el poema "De rerum natura" de Tito Lucrecio (siglo I a. C.). Cicerón (106-43 a.C.), en el ensayo "De senectute" o "Arte de envejecer", planteaba que la pérdida de memoria del "anciano" (es preciso tener en cuenta que para aquella época, la expectativa de vida media en el imperio romano era de 30 años de edad) era una enfermedad cerebral añadida al paso de los años, pero también tenía la connotación de "locura"; por lo tanto el término demencia se aplicaba a cualquier estado de deterioro cognitivo y conducta anormal incomprensible (10). Probablemente haya sido Celsius (30-50 a. C.) el primero en utilizar demencia como término médico, el cual con el paso de los años empieza a involucrar a las primeras alusiones del término de "demencia senil" en el siglo 2 d. C. propuesto por Arateous de Capadocia (en el libro III de su famosa Obra Médica), con lo cual la demencia se asociaba a un único factor de riesgo: la edad (13). Posteriormente Pablo de Egina, en su famosa publicación de Epitome, Hypomnema o Memorandum, en el siglo VII, intentó distinguir el deterioro cognitivo debido a demencia como un proceso adquirido, del que ocurre en la demencia de los procesos congénitos que acompaña al retraso mental y la oligofrenia (8).

Según Andrea Slachevsky, recién a partir de 1700 empieza a usarse, primero en Francia y posteriormente en otros países de Europa, el término de demencia para designar a estados de disfunción cognitiva con una connotación médica más precisa. En la Encyclopédie, ou Dictionnaire Raisonné des Sciences, des Arts et des Métiers, de Diderot y d'Alembert, publicada en 1765 , la demencia es definida como una "enfermedad que puede ser considerada como la parálisis del espiritu, que consiste en la abolición de la facultad de razonar. Se diferencia del delirio, que consiste en un funcionamiento aberrante de la memoria $y$ entendimiento [...]. También debe diferenciarse de la mania, que es una especie de delirio con audacia. Los signos de la demencia son fáciles de reconocer: los que padecen de una demencia son de una tal tontera que no comprenden nada de lo que se les dice, no se acuerdan de nada, no tienen ningún juicio [...]. Permanecen sin moverse [...]. La fisiología enseña que el entendimiento se realiza mediante el cambio de los estímulos que recibe la superficie o sustancia de las fibras del cerebro. La viveza de los estados del alma refleja la viveza de las impresiones sobre esas fibras [...]. La demencia se debe a una abolición de la disposición de esas fibras a reaccionar a los cambios de los estímulos. Esta abolición puede deberse a: una falla de las fibras que no pueden responder a los cambios. Sea por un defecto de conformación de estas fibras, o al efecto de alguna enfermedad o el de la vejez que alteran las fibras [...]; o a un pequeño volumen de la cabeza y aún más por escasa cantidad de cerebro; por un golpe en la cabeza que causa una alteración del cerebro; el efecto de una enfermedad incurable como la epilepsia $[. .$.$] entre otras condiciones" (9,10)$.

Por otro lado, en estas épocas aún no hay precisión del término "demencia", pero las características clínicas propias de ella son referidas como diversos cuadros que abarcaban tanto a la imbecilidad como a la idiocia, la debilidad mental y la locura. En 1771, Boissier de Sauvages, en su Nosographie Méthodique, menciona hasta 12 tipos de imbecilidad, entre las cuales destaca la imbecilidad del anciano ("l'imbécilité $d u$ vieillard"), denominada también como estado pueril, idiocia o locura senil; para Boissier, este cuadro se explica así: "por la rigidez de sus fibras nerviosas, los ancianos son menos sensibles a los estímulos externos" (14).

Posteriormente, en su Nosographie philosophique (Méthode appliqué a la médecine) de 1798, Philippe Pinel usó los términos de amentia y morosis para referirse a trastornos cognitivos, que se explican como una falla en la asociación de ideas que se manifiesta en un trastorno de la actividad, comportamiento extravagante, emociones superficiales, pérdida de memoria, dificultades en la percepción de objetos, oscurecimiento del juicio, actividad sin propósito, existencia automática y olvidos de palabras o signos para expresar las ideas. Menciona además la “demencia senil" como una forma de trastorno cognitivo adquirido en las personas de edad. Sin embargo, Pinel, no clasificó los trastornos cognitivos adquiridos y congénitos como entidades independientes (8-10).

Durante inicios del siglo XIX, el término de demencia empieza a ser usado para referirse 
principalmente a cuadros de trastornos cognitivos adquiridos (en el contexto de la búsqueda de la relación entre parálisis general progresiva y la sífilis parenquimatosa cerebral); mientras que en la segunda mitad del siglo XIX el término se restringe casi exclusivamente a trastornos irreversibles que afectan predominantemente a los ancianos. De esta manera el término demencia empieza a ser relacionado con causas secundarias, como las infecciosas (parálisis general progresiva) o a consecuencia de enfermedades crónicas. Se mantuvo una visión de síndrome de las demencias, incluyendo entre otro las demencias vesánicas, es decir, estados terminales de diferentes enfermedades mentales, como la epilepsia (11), y empieza a construirse las primeras evidencias del concepto de demencia.

Jean-Étienne Dominique Esquirol, discípulo de Pinel en el hospital de la Salpêtrière, fue uno de los primeros en propiciar una aproximación descriptiva de las demencias. Publicó en 1838 en su libro "Des maladies mentales" (15) la distinción entre demencia y retardo mental, planteándola de la siguiente manera: "El demente es como la persona que ha sido privada de su propiedad después de haber podido disfrutar de ella, es una persona rica que se ha empobrecido, el idiota siempre ha sido pobre y miserable". Junto a sus discípulos enfatizaron la heterogeneidad clínica de estos cuadros (11). Por esas mismas épocas (1826) en su publicación De la paralysie considerée chez les aliénés recherches faites dans le service de feu, LouiseFlorentine Calmeil, alumno de Esquirol, menciona las dificultades que existen para "describir las demencias, su variabilidad y matices [...] Es dificil elegir un sintoma distintivo", apoyando la "teoría dualista" de la parálisis general progresiva; diferenciando por un lado, la "demencia paralítica" y por otro lado, "el delirio". Si bien es cierto, Esquirol y otros autores se refirieron a las demencias seniles (15), fue Calmeil quien las describió de manera más acuciosa, afirmando que en las demencias seniles, "existe un compromiso constante de los órganos sensoriales, los pacientes de edad pueden estar sordos, y mostrar un trastorno del gusto, olfato, y tacto. Los estímulos externos son menos claros para ellos, tienen poca memoria de los eventos recientes, viven en el pasado, y repiten el mismo cuento; sus afectos gradualmente se desvanecen" $(9,10)$.

El primero en proponer diferentes tipos de demencia según la edad de presentación fue Guislain, quien planteó que existían dos clases de demencia: i) las demencias que afectan a las personas de edad y ii) las demencias de las personas más jóvenes (11). Pero, quizás uno de los hitos de mayor relevancia en la historia de las demencias está relacionado a AntoineLaurent-Jessé Bayle, quien en 1822 presentó un trabajo clínico-patológico en su famosa tesis (Recherches sur l'arachnitis chronique, la gastrite et la gastroentérite chronique, et la goutte, considerée comme causes de l'aliénation mentale) en la que mostraba que los síntomas físicos y mentales de la parálisis general progresiva de seis casos de enfermos del servicio de asilos de Charenton se debían a una aracnoiditis crónica, es decir, a una patología cerebral, siendo el primero en plantear la teoría de la "unicidad" (16). Pero, lo más importante, en el contexto del estudio del concepto de demencia, es que Bayle propuso el concepto de aracnoiditis crónica, propiciando una visión orgánica de las demencias, es decir, una enfermedad atribuible a lesiones en el sistema nervioso central (10).

En la segunda mitad del siglo XIX, además de identificar las demencias seniles y atribuirle un substrato neuropatológico, es decir, la presencia de lesiones en el sistema nervioso central, se proponen taxonomías de las enfermedades mentales basado en su etiología, en reemplazo de las ya existentes basadas en su sintomatología. Bénédict-Augustin Morel en su Tratado de degeneración de la especie humana publicado en 1857, por ejemplo, propuso diferenciar los trastornos mentales en seis categorías: hereditarias, tóxicas, asociadas con las neurosis, idiopáticas, simpáticas, y las demencias (17). Además, Morel sugiere que las demencias son enfermedades neurodegenerativas en las que existe una pérdida progresiva del volumen del cerebro. Para Morel, la disminución del peso del cerebro es un hecho constante en las demencias que está también presente en el envejecimiento y es una expresión de decadencia de la especie humana (11).

En la segunda mitad del siglo XIX, gracias a la elaboración del concepto de demencia y las descripciones clínicas de autores franceses, ingleses y alemanes, las demencias son definitivamente consideradas como un síndrome. Alrededor de 1900 ya se habían individualizado la demencia senil, la arterioesclerótica (reblandecimiento cerebral por isquemia crónica), las demencias infecciosas (la parálisis general), la demencia traumática y algunas de las subcorticales (por ejemplo, la enfermedad de Huntington) $(9,10,12)$. El 04 de noviembre de 1906, Alois Alzheimer en la $37^{\text {a }}$ conferencia de psiquiatras alemanes del sur-oeste, realizado en Tübingen presentó el caso de Aguste $\mathrm{D}$, paciente 
que presentó una demencia a los 51 años, publicando sus principales resultados recién un año después bajo el título "A characteristic serious disease of the cerebral cortex" en la cual describió las lesiones neuro-patológicas clásicas (placas seniles y ovillos neurofibrilares) de la demencia degenerativa (18) más frecuente, denominada luego como enfermedad de Alzheimer por Emile Krapelin (en 1910, en la $8^{\text {a }}$ edición de su Handbook of Psychiatry) $(10,18)$. Por otro lado, Arnold Pick en 1892 publicó bajo el título de "On the relationship between senile cerebral atrophy and aphasia" el caso de Augusto $\mathrm{H}$ de 71 años de edad del Instituto Psiquiátrico de Praga, quien inusualmente presentó al inicio un severo desorden del lenguaje con rápido deterioro cognitivo y funcional. Luego de la muerte de Augusto $\mathrm{H}$, la autopsia reveló severa atrofia cerebral con predominio del lóbulo temporal izquierdo; pero Pick no logró realizar estudios histopatológicos; sin embargo llama la atención sobre la focalización de algunos tipos de demencia (19). Recién en 1911 Alois Alzheimer describió los cuerpos y células de Pick, los marcadores típicos de la demencia fronto-temporal (8$10,14)$.

El concepto actual de demencia se construyó a finales del siglo XIX y a principios del siglo XX bajo un claro y definitivo paradigma cognitivo: las demencias son el resultado de trastornos irreversibles de las funciones intelectuales $(9,10,12)$. Sin embargo, este planteamiento, limitó el estudio de los trastornos no cognitivos de las demencias; pero sobre todo, el estudio de la interfase entre demencias y enfermedades psiquiátricas. No obstante, en las dos últimas décadas se ha avanzado en la comprensión de los trastornos no cognitivos de las demencias, como es el caso de las demencias frontotemporales, cuyos principales síntomas son trastornos de la conducta $(10,14)$.

Conforme avanzaron las neurociencias, se planteó la necesidad de perfilar unos criterios diagnósticos de consenso. En 1980 se publicaron los criterios del Manual diagnóstico y estadístico de las enfermedades mentales, en su tercera versión (DSM-3), que incluyó los primeros criterios operativos para el diagnóstico de los trastornos mentales, incluidas las demencias; sin embargo, la definición que usó fue una descripción general del síndrome de demencia y sus posibles causas, sin proponer en forma explícita ningún criterio operativo para el diagnóstico, es decir, formalmente, los criterios del síndrome de demencia no existían aún. Recién en 1984 los criterios del National Institute of Neurological and Communicative Disorders and Stroke and the Alzheimer's Disease and Related
Disorders Association, NINCDS-ADRDA define a la demencia como "el declive de las funciones cognitivas en comparación con el nivel previo del funcionamiento del paciente determinado por la historia de declive y por las alteraciones apreciadas en el examen clínico y mediante pruebas neuropsicológicas" (20), de esta manera la demencia podría corresponder a un síndrome clínico (tal vez, demencia semántica), a un síndrome anatomo-clínico (quizá, demencia subcortical), a un síndrome anatomo-patológico (como la demencia vascular), a un grupo de patologías caracterizadas según su evolución (como las demencias reversibles), a entidades patológicas definidas (en este caso, demencia de la enfermedad de Alzheimer) o a entidades con una etiología precisa (por ejemplo, demencia por neurosífilis). Sin embargo, los más utilizados fueron los criterios del DSM-4 (21) y de la Clasificación Internacional de Enfermedades, décima edición (CIE10), que son parte de la clasificación internacional de enfermedades publicada por la OMS (22), que se refirieron a demencia como "deterioro de la memoria (capacidad de aprender nueva información o recordar información previamente aprendida); con una o más alteraciones cognitivas como afasia, apraxia, agnosia o de las funciones ejecutivas; lo suficiente grave para provocar un deterioro significativo de la actividad social o laboral; que representan un déficit respecto a un nivel previo; que no aparecen durante un síndrome confusional agudo; con evidencia clínica o mediante pruebas complementarias de que estas alteraciones no son debidas a causa orgánica o al efecto de una sustancia tóxica". Pero, ambas propuestas tuvieron importantes limitaciones. Por una parte, los criterios DSM-4 se crearon basándose en la demencia tipo Alzheimer y no reflejan el conjunto de las demencias. De hecho, los criterios del DSM-4 consideran el trastorno de memoria como el principal síntoma de las demencias, lo que, si bien es válido para EA, no se ajusta al patrón de presentación de otras demencias en las que la memoria está preservada, como las demencias fronto-temporales o la demencia vascular. De la misma manera, los criterios del CIE-10 se enfocan en los desórdenes de la memoria, aunque precisa la naturaleza progresiva de la deficiencia de múltiples funciones cognitivas; pero falla en no considerar los estadios preclínicos previos a la demencia, como el deterioro cognitivo leve.

El DSM-5 modificó sustancialmente sus criterios con respecto a la versión anterior (23). Al ser una clasificación de enfermedades tampoco incluye los estadios preclínicos, pero si contempla un estadio patológico pre-demencia similar al deterioro cognitivo 
leve propuesto por Petersen e introduce el concepto de "trastorno neurocognitivo" (24), que ocupa el lugar de los "trastornos mentales orgánicos" de ediciones anteriores. Según el DSM-5, los trastornos neurocognitivos se dividen en tres categorías: delirium, trastorno neurocognitivo menor y trastorno neurocognitivo mayor. El trastorno neurocognitivo mayor y el menor se diferencian en función de la intensidad de los síntomas y su repercusión en la funcionalidad del paciente (23). De la misma manera que las versiones previas, el DSM-5 no hace referencia a los síntomas psicológicos y conductuales de demencia dentro de los criterios diagnósticos, pese a la evidencia de que esos síntomas pueden ser los primeros en aparecer en otros tipos de demencia (25). Además, el DSM-5 no contempla las posibilidades etiológicas "mixtas", lo cual contradice a lo que pasa en el "mundo real" en el que la evidencia acumulada refleja relativa escasez de casos de etiología "pura".

Los nuevos criterios diagnósticos para EA postulados por los grupos de trabajo de Bruno Dubois y el National Institute of Aging (NIA) estadounidense con la Alzheimer's Association (AA), conocidos como los criterios NIA-AA han tratado de superar el planteamiento de los criterios NINCDS-ADRDA acerca de la unión inseparable de la patología neuronal y clínica de la demencia $(26,27)$. Uno de los principales pasos adelante ha sido la consideración de la EA como un proceso nosológico que posee una continuidad desde fases asintomáticas hasta el desarrollo de un cuadro demencial completamente caracterizado. De esta manera, existirían estadios asintomáticos, oligosintomáticos y pluri-sintomáticos, que formarían parte del mismo proceso patológico. También es importante porque llama la atención acerca de la posibilidad de utilizar biomarcadores que permitirían el diagnóstico en fases asintomáticas. El grupo de trabajo dirigido por el profesor Bruno Dubois se refiere exclusivamente a la EA, que la define sobre la base de la evaluación del déficit mnésico característico de la enfermedad y el apoyo en los biomarcadores, entre los cuales considera: evidencia de depósito de $\beta$-amiloide (puesto de manifiesto a través de los resultados, como niveles bajos de proteína $A \beta 42$ en líquido cefalorraquídeo o amiloide positivo en tomografía por emisión de positrones-PET), evidencia de lesión o degeneración neuronal progresiva (como la presencia de niveles incrementados de proteínas tau total y tau fosforilada en líquido cefalorraquídeo), captación disminuida de fluorodeoxiglucosa en la corteza parieto-temporal en estudios de PET, o atrofia desproporcionada en los lóbulos temporal medio, basal y lateral y en el lóbulo parietal medio en imágenes por resonancia magnética (IRM). Para este grupo de trabajo, sin biomarcadores positivos no se puede diagnosticar EA, con independencia del estadio en que se encuentre (26).

\section{Criterios actuales para el diagnóstico de las diferentes formas de demencia}

\section{Criterios actuales en el diagnóstico de demencia}

El Manual diagnóstico y estadístico de las enfermedades mentales (DSM) de la American Psychiatric Association (APA) en su quinta versión (DSM-5) introduce el concepto de 'trastorno neurocognitivo', que ocupa el lugar de los 'trastornos mentales orgánicos' de ediciones anteriores (23). Así, los trastornos neurocognitivos se dividirán en tres categorías: delirium, trastorno neurocognitivo menor (para referirse a deterioro cognitivo leve) y trastorno neurocognitivo mayor, el cual conocemos como demencia. Establece que los dominios sintomáticos estudiados para el diagnóstico serán: atención, función ejecutiva, aprendizaje, memoria, lenguaje, funciones visuo-perceptivas y visuo-constructivas y cognición social. El trastorno neurocognitivo mayor y el menor se diferencian básicamente en función de la intensidad de los síntomas y su repercusión en la funcionalidad del paciente. Los criterios diagnósticos para el trastorno neurocognitivo mayor requieren: 1) la evidencia de un declive cognitivo sustancial en relación a un nivel previo de mayor desempeño en uno o más de los dominios cognitivos, referidos como preocupación o quejas del individuo, de un tercero informado (cuidador), o del facultativo con respecto a un declive sustancial en las funciones cognitivas; 2) declive en el desempeño neuropsicológico, implicando un desempeño en las pruebas cognitivas del rango de dos o más desviaciones estándares por debajo de lo esperado en la evaluación neuropsicológica normada y validada ante una evaluación clínica equivalente; 3) que interfieran con la independencia; y 4) que no ocurran exclusivamente en el contexto de delirium $(23,25)$.

\section{Definiendo el estadio pre-demencia}

Es el trastorno neurocognitivo menor del DSM5 , conocido previamente como deterioro cognitivo leve (DCL). Se puede definir como aquel deterioro cognitivo que supera lo normalmente esperable para la edad, pero que no cumple con criterios de demencia, pues la funcionalidad está aún preservada (28). Hasta antes del 2001 existían diversos criterios, pero los 
del International Working Group on Mild Cognitive Impairment fueron los más utilizados (29), y debían reunir los siguientes criterios: 1) problemas de memoria, particularmente si son corroborados por un informante; 2) déficit de memoria objetivado mediante evaluación neuropsicológica $(1,5$ desviaciones estándar (DE) por debajo de lo esperado para edad y escolaridad); 3) preservación de la función cognitiva general; 4) conservación de las actividades de la vida diaria; y, 5) ausencia de demencia. Según este grupo de trabajo el DCL podría ser de dominio único o de multi-dominio, siendo el de dominio único de tipo amnésico el que tiene mayores probabilidades de progresar hacia EA (28). Posteriormente, el profesor Dubois del hospital de La Salpetriere propuso criterios para el diagnóstico de demencia de la EA, no aplicables para otro tipo de demencia (26). En esta propuesta, lo importante desde el punto de vista clínico es la precisión en el dominio cognitivo afectado, y precisa que se produce una afectación gradual y progresiva de la memoria episódica, pudiendo ser aislada o asociada con otras alteraciones cognitivas, pero siempre evidenciada por los resultados de las pruebas neuropsicológicas. A este hallazgo clínico se propone la asociación de biomarcadores, como la atrofia en el lóbulo temporal medial evidenciada por IRM, la alteración de los biomarcadores en líquido cefalorraquídeo, alteraciones en pruebas de neuroimagen funcional con PET o una mutación autosómica dominante en un familiar de primer grado. Esta clasificación difiere de la que clínicamente se había venido utilizando a raíz de la introducción del término 'deterioro cognitivo leve'. Si previamente a la publicación de los criterios del grupo de Dubois el deterioro cognitivo leve era una afectación cognitiva que excedía el declive cognitivo asociado a la edad y que podía degenerar en un cuadro demencial, tipo Alzheimer o no, según estos nuevos criterios, el empleo del término 'deterioro cognitivo leve' debe reservarse para cuando el deterioro incipiente -por la ausencia de bio-marcadores o de déficit específico de memoria episódica- no puede atribuirse a una EA. Es decir, la EA en grado incipiente o inicial puede producir un deterioro cognitivo leve, que ya debe recibir el nombre de EA aunque aún no haya producido demencia. Sólo en el caso de que este deterioro leve no sea causado por una EA, recibe el nombre de 'deterioro cognitivo leve', según el grupo de Dubois $(26,28)$. Para resumir,
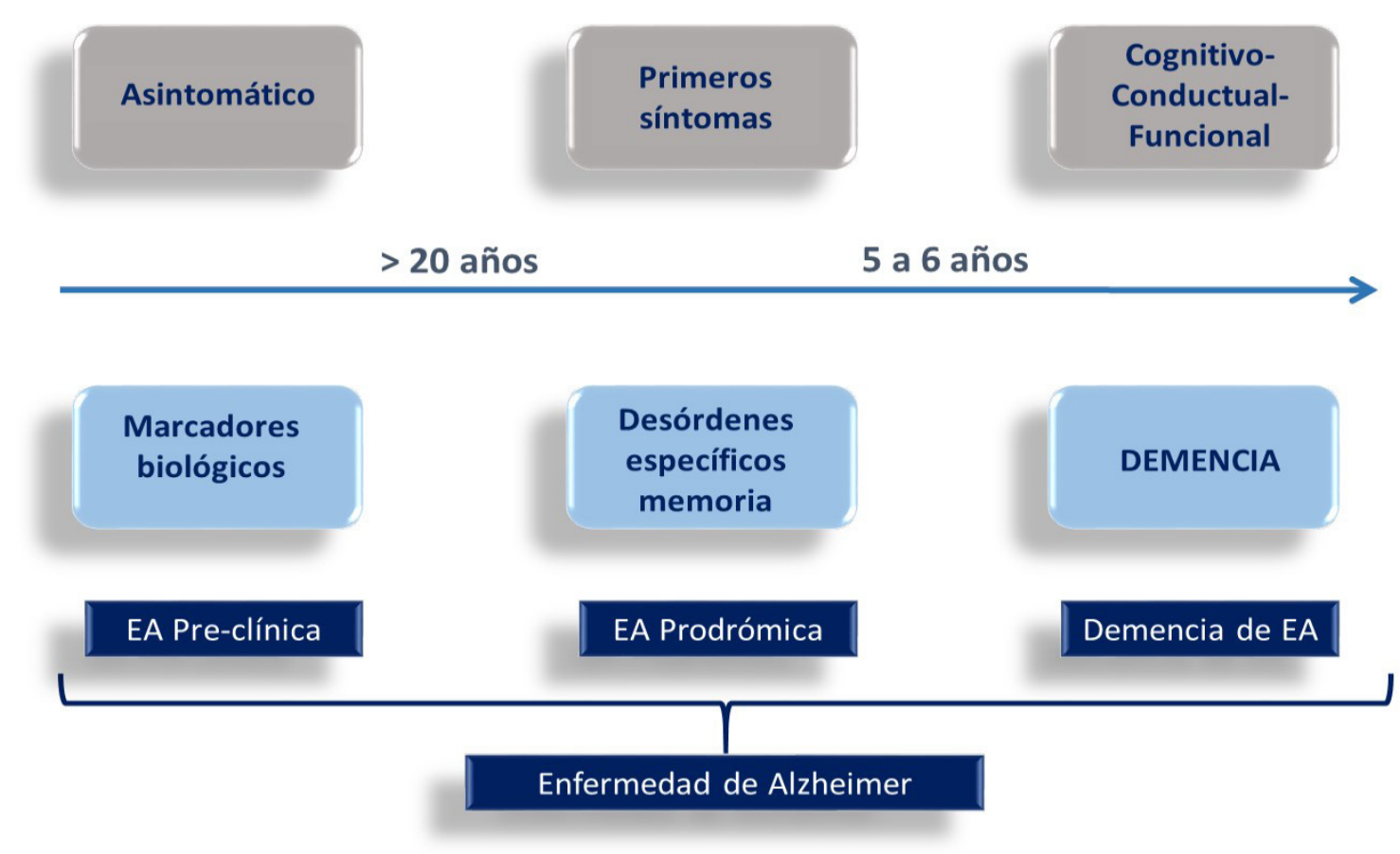

EA: Enfermedad de Alzheimer

*Modificado de Dubois B, Feldman HH, Jacova C, Cummings J, DeKosky ST, Barberger-Gateau P, et al. Revising the definition of Alzheimer's disease: a new lexicón. Lancet Neurol 2010; 9(11): 1118-1127.

Gráfico 1. Evolución de enfermedad de Alzheimer desde el depósito de las placas seniles y ovillos neurofibrilares. 
según el profesor Dubois, la EA es un continum que inicia con el depósito de placas de amiloide y ovillos neurofibrilares, durante el cual se denomina EA preclínica; que evolucionará en el curso de los años hacia EA prodrómica, cuando empiezan los primeros problemas de memoria episódica, y posteriormente llega a demencia de EA (cuando se compromete la funcionalidad), en sus diversos estadios: leve, moderado y severo (gráfico 1).

Recientemente, el NIA-AA, propone al DCL como un síndrome definido por criterios clínicos, cognitivos y funcionales; asimismo, otras causas adicionales pueden coexistir en un individuo que reúne los criterios de DCL, por lo que se pone especial énfasis a los datos clínicos, y otorga un valor adicional a los biomarcadores (beta-amiloide 42, tau/tau fosforilado en LCR e imágenes cerebrales), pero, secundario y dependiente de los datos clínicos (30). La comparación entre los criterios de Dubois y los criterios NIA-AA para establecer el diagnóstico de EA muestra una aproximación más clínica por parte de estos últimos, en los cuales se concede gran importancia al examen neuropsicológico de diversos ámbitos cognitivos, a la exploración psicopatológica y a la valoración funcional, y no sólo a la alteración del nivel de memoria. Ello permite aproximarse al diagnóstico en el marco de la clínica habitual, sin necesidad de la gran sofisticación tecnológica necesaria para la detección de biomarcadores, que los criterios del profesor Dubois requieren. Para los criterios NIA-AA, la presencia de biomarcadores apoya el diagnóstico, pero su empleo no es imprescindible. Estos criterios conservan además el concepto de deterioro cognitivo leve y definen los criterios para diagnosticar una demencia, sea o no debida a EA.

Por otro lado, desde la perspectiva clínica, DCL debe diferenciarse del deterioro de la memoria del envejecimiento normal, referido como 'deterioro de memoria asociado a edad' (DMAE). El National Institute of Mental Health (NIMH) de Estados Unidos propuso criterios diagnósticos para el DMAE: 1) edad mayor de 50 años; 2) existencia de quejas subjetivas de pérdida de memoria, descrita como gradual, sin empeoramiento brusco ni ocurrida en meses recientes, reflejadas en la vida cotidiana como dificultad para recordar nombres de personas conocidas; 3) rendimiento en las pruebas de memoria de por lo menos $1 \mathrm{DE}$ por debajo del promedio establecido para adultos jóvenes en la prueba estandarizada de memoria secundaria (memoria reciente); 4) conservación de las otras funciones intelectuales; y, 5) ningún criterio para demencia o cualquier otra condición médica que pueda causar déficit cognitivo $(28,31)$.

\section{Criterios diagnósticos de demencia de la enfermedad de Alzheimer}

El profesor McKhan en 2011 actualizó los criterios del National Institute of Neurological and Communicative Disorders and Stroke and the Alzheimer's Disease and Related Disorders Association (NINCDS-ARDRA) de 1984 de su misma autoría. Esta propuesta surge del trabajo conjunto de grupos pertenecientes al National Institute of Aging estadounidense (NIA) y la Alzheimer's Association (AA), conocidos ahora como los criterios del NIAAA (27) desde una perspectiva clínico-biológica. Los criterios de demencia debido a EA probable exigen el cumplimiento estricto de los criterios nucleares (cuadro clínico de inicio insidioso, historia clara de un empeoramiento cognitivo progresivo referido $\mathrm{u}$ observado y el déficit inicial y más prominente que puede seguir un patrón de presentación amnésicoacompañado de afectación en al menos otro dominio cognitivo, o no amnésico-trastorno del lenguaje, trastorno visuo-perceptivo o disfunción ejecutiva) y, además, la ausencia de evidencia de enfermedad cerebrovascular, demencia con cuerpos de Lewy, demencia fronto-temporal en todas sus variables o de otras enfermedades, o consumo de sustancias que puedan afectar a la cognición de forma sustancial. Estos criterios del NIA-AA permiten agregar el término "con evidencia de procesos fisiopatológicos de EA" en caso de que sea posible realizar un estudio de bio-marcadores. Este es el caso cuando, a una demencia debida a EA posible o probable, se le puede añadir la presencia de bio-marcadores, tales como: evidencia de depósito de $\beta$-amiloide (puesto de manifiesto a través de los resultados, como niveles bajos de proteína $A \beta 42$ en LCR o amiloide positivo en tomografía por emisión de positrones-PET), evidencia de lesión o degeneración neuronal progresiva (como la presencia de niveles incrementados de proteínas tau total y tau fosforilada en líquido cefalorraquídeo), captación disminuida de fluorodeoxiglucosa en la corteza parieto-temporal en estudios de PET, o atrofia desproporcionada en los lóbulos temporal medio, basal y lateral y en el lóbulo parietal medio en IRM. Por último, recomiendan emplear el término "demencia debida a EA fisiopatológicamente probada" cuando se cumplen criterios clínicos y anatomo-patológicos de EA. El término "demencia improbablemente debida a EA" se aplicará cuando el proceso no cumple criterios de EA, cuando existe suficiente evidencia de otro 
diagnóstico neurodegenerativo, de origen médico o por sustancias, o ante un estudio de bio-marcadores negativo para la EA $(27,28,31)$.

Recientemente, en abril de 2018, el NIAAA ha publicado una revisión de los criterios del 2011, haciendo énfasis en que estas nuevas recomendaciones son de exclusiva aplicación en el contexto de investigación clínica (estudios de cohorte longitudinal y ensayos clínicos aleatorizados controlados con placebo), pero no aplicables para la práctica clínica diaria. La principal propuesta es que el diagnóstico de EA no depende de los síntomas clínicos (el perfil de deterioro cognitivo leve amnésico no es específico de EA, y por otro lado ciertos perfiles no amnésicos también pueden desarrollar EA, como los síntomas visuales complejos de la atrofia cortical posterior o los problemas del lenguaje de las afasias primarias progresivas), sino de la presencia de los biomarcadores, por lo que plantean una exclusiva perspectiva biológica. Se plantean tres grupos de biomarcadores, etiquetados como A ( $\beta$-amiloide), $\mathrm{T}$ (Tau patológico) y $\mathrm{N}$ (neurodegeneración/injuria neuronal), que se conocen como el sistema ATN. Los biomarcadores para placas de $\beta$-amiloide (etiquetados como A) se miden a través de marcadores que se unen al $\beta$-amiloide cortical mediante PET o bajas concentraciones de $\beta-A_{42}$ medidos en LCR; los biomarcadores para Tau patológico o fosforilado (etiquetados como T) se miden a través de marcadores que se unen al Tau cortical mediante PET o altas concentraciones de Tau fosforilado (P-tau) medidos en LCR; y los biomarcadores para neurodegeneración/ injuria neuronal (etiquetados como $\mathrm{N}$ ) se miden a través del hipometabolismo de glucosa mediante PET o altas concentraciones de Tau total medido en LCR o atrofia medidas en IRM cerebral. Así se establecen perfiles ATN de biomarcadores (8 posibles: A-T-N-, $\mathrm{A}+\mathrm{T}-\mathrm{N}-, \quad \mathrm{A}+\mathrm{T}+\mathrm{N}-, \quad \mathrm{A}+\mathrm{T}+\mathrm{N}+, \quad \mathrm{A}+\mathrm{T}-\mathrm{N}+, \quad \mathrm{A}-\mathrm{T}+\mathrm{N}-$, $\mathrm{A}-\mathrm{T}-\mathrm{N}+$ y $\mathrm{A}-\mathrm{T}+\mathrm{N}+$ ) y categoría de biomarcadores (desde biomarcadores negativos para EA, pasando por el continum de EA, hasta cambios patológicos no relacionados a EA).

De esta manera, podemos establecer que una persona viva tiene EA sí presenta al menos evidencia de $\beta$-amiloide; sin embargo, se requiere evidencia de tanto $\beta$-amiloide como Tau fosforilado para

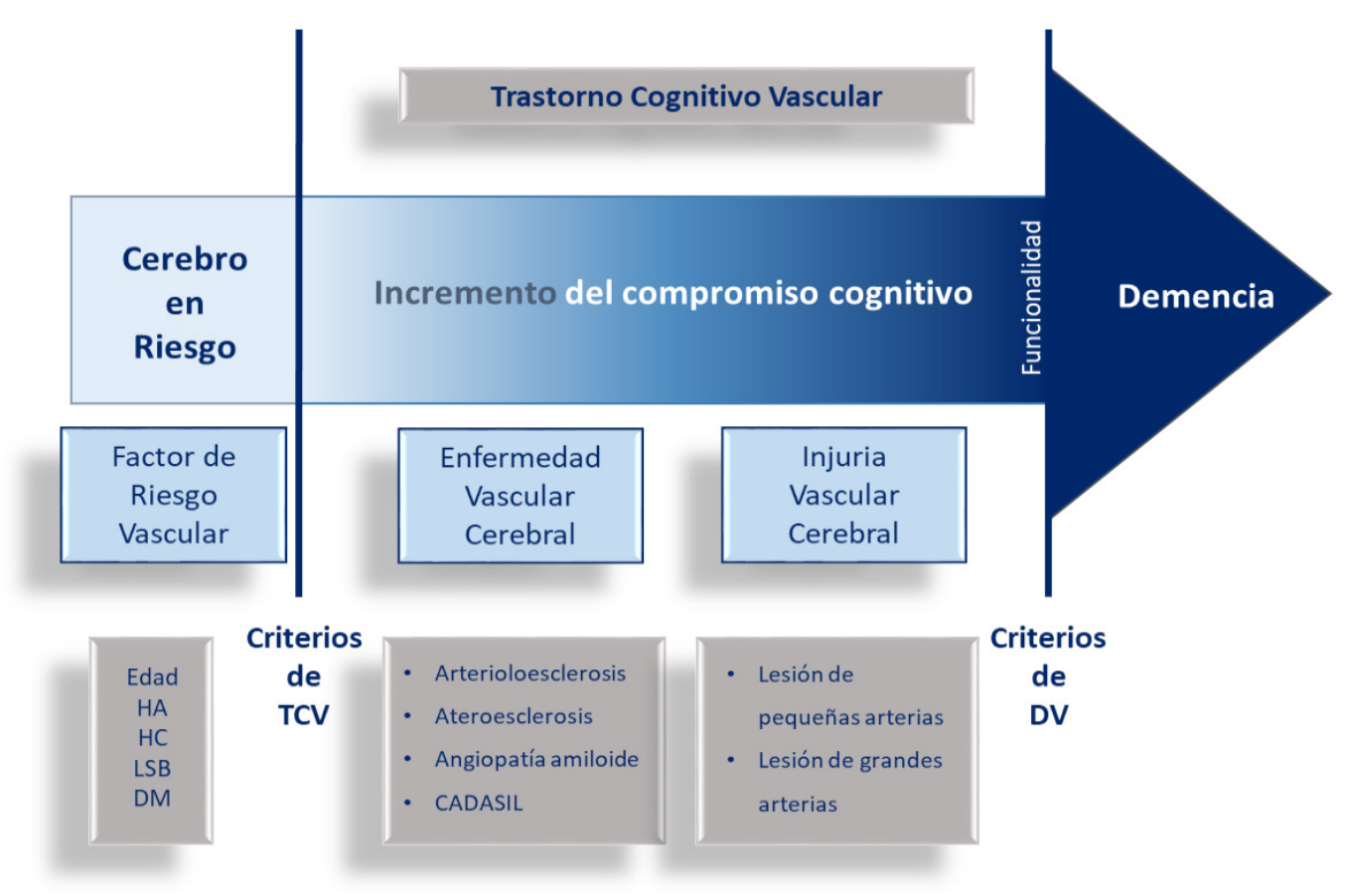

TCV: trastorno cognitivo vascular; DV: demencia vascular; HA: hipertensión arterial, HC: homocisteína; LSB: lesión de sustancia blanca; DM: diabetes mellitus.

*Modificado de Bowler JV. Modern concept of vascular cognitive impairment. British Medical Bulletin 2007; 83: 291-305.

Gráfico 2. Evolución del trastorno cognitivo vascular desde la aparición de los factores de riesgo vascular hasta el compromiso de la funcionalidad. 
completar los criterios neuropatológicos, mientras que la evidencia de neurodegeneración/injuria axonal y la información cognitiva establecen severidad de la enfermedad (7).

\section{Criterios del diagnóstico de demencia vascular}

Según los conservadores criterios del National Institute of Neurological Disorders and StrokeAssociation Internationale pour la Recherche et l'Enseignment en Neurosciences (NINDS-AIREN), para definir demencia vascular (DV) era necesario demostrar evidencia de compromiso de la memoria y al menos dos otras áreas cognitivas, además de evidencia de lesión vascular cerebral (signos neurológicos focales e infartos/lesión de sustancia blanca en las imágenes cerebrales) y que el inicio de la demencia se desarrolle dentro de los 3 meses de producido el ictus cerebral (32); sin embargo, la gran limitación de dicha clasificación era la disfunción de memoria como principal característica, dominio cognitivo que se afecta sólo en estadios avanzados, y que además desde el punto de vista clínico sugería enfermedad de Alzheimer.

En el contexto actual, DV puede ser definida como deficiencia del control ejecutivo que origina pérdida de la función de las actividades de vida diaria instrumentales, sin necesidad de evidencia clínica de lesión neurológica focal, pero que exige la evidencia de lesión de sustancia blanca, pudiendo esta ser focal o difusa, con o sin infarto (33). Los criterios diagnósticos de DV incluyen: 1) declinación de las funciones cognitivas, con evidencia de un deterioro previo o una variación en las evaluaciones basales previas en más de 2 dominios cognitivos que sean lo suficientemente severos para afectar las actividades de la vida diaria (AVD); 2) que esté basado en pruebas cognitivas y por lo menos cuatro dominios evaluados: funciones ejecutivas/atención, lenguaje, memoria y funciones visuo-espaciales; 3) que los déficits de las AVDs sean independientes de las secuelas motoras/sensoriales del evento vascular. Además, para el diagnóstico de DV probable se busca establecer una clara relación entre el evento vascular y el inicio del deterioro cognitivo, o una clara relación entre la severidad y el patrón del deterioro cognitivo con la presencia de enfermedad vascular cerebral (EVC) subcortical o difusa, sin necesidad de evidencia clínica de lesión neurológica focal y que en imágenes por resonancia magnética (IRM) de cerebro, se evidencie por lo menos algunas lesiones de sustancia blanca (LSB), pudiendo ésta ser focal o difusa, con o sin infarto evidente. Las LSB en áreas específicas subcorticales y del lóbulo temporal son la clave del diagnóstico de una forma genética de DV en individuos jóvenes, conocidas como cerebral autosomal dominant arteriopathy with subcortical infarcts and leukoencephalopathy (CADASIL) y también en algunos pacientes con desórdenes autoinmunes (lupus eritematoso sistémico y sindrome de Sjögren); sin embargo debe quedar claro que LSB podría observarse en individuos sanos de la tercera edad, así como en pacientes con EA. De tal manera, que en el nuevo enfoque, DV es un término arbitrario y tardío en la evolución de la EVC, que estaría al final del continuum de una serie de sucesos originados por los factores de riesgo vascular cerebral $(34,35)$.

\section{El trastorno cognitivo vascular como estadio de pre- demencia vascular}

El trastorno cognitivo vascular (TCV) agrupa todos los estadios donde el compromiso cognitivo puede ser atribuido a EVC y sea mayor a lo esperado para el envejecimiento normal; mientras que DV vendría a ser el estadio final del TCV, donde el compromiso cognitivo es lo suficientemente severo para interferir con el funcionamiento social y ocupacional (36) (gráfico 2). La finalidad de poner énfasis en definir TCV es llamar la atención en la prevención y tratamiento oportuno de tal vez el único estado pre-demencia que realmente puede evitar la progresión hacia un estado de demencia, la DV.

El TCV es un síndrome o fenotipo causado por EVC. A diferencia de EA, TCV no es por sí mismo una enfermedad, es un conjunto de síndromes que representan una injuria vascular cerebral (IVC), y además la EVC en la mayoría de casos podría progresar a DV, mientras que EA invariablemente progresa hacia demencia de la EA. Para resumir, TCV se origina con la aparición de los factores de riesgo vascular (edad, hipertensión arterial, hiper-homocisteinemia, e hiper-señales de sustancia blanca, conocidas también como hiper-intensidades de sustancia blanca o lesiones de sustancia blanca), los cuales generan EVC (arterioloesclerosis, ateroesclerosis, angiopatia amiloide y CADASIL-Cerebral autosomal dominant arteriopathy with subcortical infarcts and leukoencephalopathy) y finalmente injuria vascular cerebral (IVC), que va a depender del tamaño de la arteria comprometida, por lo tanto tendremos lesión de grandes arterias y lesiones de pequeñas arterias), la cual según su localización genera diversos tipos de compromiso cognitivo (35). 


\section{Criterios actuales en el diagnóstico de demencia fronto-temporal}

Tuvieron que pasar más de 13 años para que en el 2011, Rascovsky (37) y Gorno-Tempini et al., (38) replantearan los criterios de diagnóstico clínico de la antiguamente llamada enfermedad de Pick o degeneración fronto-temporal, propuesta por Neary et al., en 1998 (39). En el momento actual se reconoce como demencia fronto-temporal (DFT) y comprende dos síndromes claramente definidos según síntomas clínicos y el patrón regional de atrofia cerebral: el primero, caracterizado por el predominio de síntomas conductuales se denomina demencia fronto-temporal, variante conductual (DFTvc) $(37,40)$; y el segundo, caracterizado por el predominio del compromiso del lenguaje, se denomina afasia progresiva primaria (APP) (38), e incluye a la afasia progresiva no fluente (APNF), demencia semántica (DS), y la afasia logopénica (AL).

Los pacientes con DFTve frecuentemente son mal diagnosticados como portadores de trastornos psiquiátricos $\mathrm{u}$ otras enfermedades neurológicas, porque el inicio de su presentación clínica involucra cambios conductuales y de la personalidad $(40,41)$, mientras que desde el punto de vista neuropsicológico, el compromiso de la memoria se presenta en estadios avanzados, siendo el compromiso de las funciones ejecutivas la característica principal en los estadios iniciales. Los criterios del consorcio internacional para DFTve (37), distinguen con claridad las categorías posible, probable y definitiva. Tres de un grupo de 6 síntomas tienen que ser positivos para alcanzar la categoría diagnostica de DFT conductual posible. Estos grupos de síntomas son más intuitivos, fáciles de memorizar y sobre todo, fáciles de aplicar y usar en la práctica clínica diaria. Ellos están referidos a dominios globales de la cognición social, el funcionamiento cognitivo y conductual. Mientras que en los anteriores criterios de Neary (39) los hallazgos en las imágenes cerebrales eran una característica de soporte, a partir de 2011 los hallazgos positivos en las imágenes cerebrales son obligatorios para un diagnóstico de DFT conductual. Por otro lado, un nuevo elemento clave en estos criterios es la exclusión de los casos de DFT conductual probable, cuando se demuestra los resultados de un biomarcador para EA, basado en el hallazgo en LCR o una imagen de amiloide positivo en PET.

Los síntomas cognitivo/conductuales que deben estar presentes para calificar como DFTve posible (al menos 3 de los 6, y el término "temprano" se refiere a que los síntomas deben estar presentes dentro de los 3 primeros años) son: inicio temprano de desinhibición conductual (pudiendo ser uno de los siguientes: conducta social inapropiada, pérdida de las maneras o el decoro, actos impulsivos menores), inicio temprano de apatía o inercia, inicio temprano de pérdida de simpatía o aparición de apatía (puede ser uno de los siguientes: respuesta disminuida a las necesidades y sentimientos de los demás, disminución del interés social o de la calidez personal), inicio temprano de conducta perseverante, estereotipada, ritualista/compulsiva (puede ser uno de los siguientes: movimientos repetitivos simples, conductas compulsivas o ritualistas complejas, estereotipia del habla), hiper-oralidad y cambios en la dieta (se refiere a uno de los siguientes: preferencias por ciertos alimentos, "atracones" de comida, consumo excesivo de alcohol/cigarrillos, exploración oral o consumo de objetos no comestibles), y perfil neuropsicológico con preservación de las funciones de memoria y viso-espaciales (37). Se considera DFTvc probable, cuando cumple criterios de DFTvc posible, además de declinación funcional significativa y atrofia frontal y/o temporal anterior en la IRM, o hipo-perfusión o hipo-metabolismo en frontal y/o temporal anterior en PET o SPECT. Para DFTve definitiva, debe cumplir con los criterios de DFTve probable o posible y evidencia histopatológica en la biopsia o post-morten, o presencia de una mutación patogénica desconocida. En todos los supuestos diagnósticos debe cumplir con los siguientes criterios de exclusión: los patrones de la deficiencia son mejor explicadas por otro desorden médico o del sistema nervioso no degenerativo, los disturbios conductuales son explicados mejor por un diagnostico psiquiátrico y los biomarcadores son claramente indicativos de EA u otro proceso neurodegenerativo $(37,42)$.

\section{Criterios actuales en el diagnóstico de demencia con cuerpos de Lewy}

El Consorcio para el diagnóstico de demencia con cuerpos de Lewy (DCLewy) ha publicado, después de 12 años, el cuarto reporte de los criterios revisados para el diagnóstico clínico y patológico de DCLewy (43), dado que los cambios realizados en el tercer reporte (44) incrementaron la sensibilidad diagnóstica, pero no la tasa de detección en la práctica clínica, donde la mayoría de casos seguían siendo diagnosticados como EA. Los criterios han sido modificados para distinguir claramente entre características clínicas y bio-marcadores diagnósticos. Los síntomas y signos 
clínicos se ponderan como características nucleares o de soporte y los bio-marcadores como características sugestivas o de soporte. Aunque tienen menos peso diagnóstico, las características de soporte son valiosas en la toma de decisiones clínicas, actuando como indicativos o añadiendo evidencia para el diagnóstico de DCLewy. La categoría de características sugestivas del tercer reporte (44) ha sido eliminada, por lo que el desorden conductual del sueño REM (DCSREM), hipersensibilidad a neurolépticos y la baja captación del transportador de dopamina en imágenes cerebrales ha sido reasignado. Los criterios revisados generan categorías de DCLewy probables y posibles, similares al reporte previo (43).

El requisito esencial para el diagnóstico de DCLewy es la presencia de demencia, que se caracteriza por declinación cognitiva progresiva de suficiente magnitud para interferir con el funcionamiento normal social u ocupacional (es decir deterioro de las actividades de vida diaria). Las características clínicas nucleares (Fluctuación en la cognición, alucinaciones visuales recurrentes, y características espontáneas de parkinsonismo) se presentan en estadios tempranos y puede persistir a través del curso de la enfermedad. Las características clínicas de soporte pueden presentarse en cualquier momento, incluso en etapas tempranas, pero en forma aislada tienen baja especificidad. Se agregan a la lista dos signos clínicos: hipersomnia (somnolencia diurna excesiva) e hiposmia (disminución de la olfación), los cuales pueden ocurrir en estadios más tempranos en DCLewy comparados con EA. Los episodios transitorios de pérdida de conciencia, "ausentismo" o "ensimismamiento" (falta de respuesta) pueden representar una forma extrema de fluctuación cognitiva, difícil de distinguir del verdadero síncope. La severa sensibilidad a neurolépticos se inserta ahora como característica de soporte, debido a que la disminución de la prescripción de antipsicóticos bloqueadores de los receptores D2 en DCLewy limita su utilidad diagnóstica. Si uno o más de los bio-marcadores sugestivos se encuentran presentes, asociado con una o más de las características clínicas nucleares, entonces podemos establecer un diagnóstico de DCLewy probable. Si encontramos criterios de demencia, sin ningún criterio clínico nuclear, pero con uno o más criterios de bio-marcadores sugestivos, podemos clasificar como un caso de DCLewy posible. Ningún caso de DCLewy probable puede ser realizado solo sobre la base de la presencia de bio-marcadores. Los bio-marcadores sugestivos a considerar son disminución de la captación de transportadores de dopamina (DAT) en los ganglios basales, demostrado por imágenes cerebrales en SPECT o PET; disminución de la captación de ${ }^{123}$ I-metayodobenzilguanidina ( $\left.{ }^{123} \mathrm{I}-\mathrm{MIBG}\right)$ en la gammagrafía miocárdica y confirmación del sueño REM sin atonía (DCSREM) en la polisomnografía (PSG). Los bio-marcadores de soporte que ayudan a la evaluación diagnóstica, pero sin una clara especificidad diagnóstica, incluyen: relativa preservación en estructura del lóbulo temporal medial medidos por IRM/TC cerebral, hipo-metabolismo o hipo-perfusión de predominio occipital y cíngulo posterior medidos por imágenes cerebrales de PET/ SPECT, y significativas ondas lentas a nivel posterior con fluctuaciones periódicas en el rango pre-alfa/theta medido en la actividad electroencefalográfica (43).

\section{Criterios actuales en el diagnóstico de demencia mixta}

No hay consenso respecto a los criterios clínicos para el diagnóstico de demencia mixta (DMx), lo cual pone en duda la existencia de esta entidad clínica (45). La clasificación del Consortium to Establish a Registry for Alzheimer's Disease (CERAD) no considera DMx en su clasificación (46), y los criterios del Alzheimer's Disease Diagnostic and Treatment Centers (ADDTC) exigen la evidencia de una EVC estrechamente relacionada a demencia (referida sin precisar el tiempo), además de la patología típica de EA (47). Por su parte, los criterios del National Institute of Neurological Disorders and Stroke and Association Internationale pour la Recherche' et l'Enseignement en Neurosciences (NINDS-AIREN) sugieren demostrar evidencia de compromiso de memoria y al menos dos otras áreas cognitivas, además de evidencia de lesión vascular cerebral (signos neurológicos focales e infartos/lesión de sustancia blanca en las imágenes cerebrales) y que el inicio de la demencia se presente dentro de los 3 meses de producido el ictus cerebral (32). En el 2011, la American Heart Association y la American Stroke Association (AHA/ ASA) reconocen que tanto la patología vascular y la degenerativa son desórdenes frecuentes y coexistentes en la tercera edad y que cada uno separadamente adiciona al otro la posibilidad de desarrollar deterioro cognitivo y demencia produciendo superpuestos fenotipos clínicos y de neuroimágenes. En ese sentido, Gorelick y colaboradores en su publicación de Stroke del 2011 plantean la necesidad de resolver inquietudes no satisfechas para discriminar demencia vascular de EA, mejorando el conocimiento de la relación entre los estudios de neuroimágenes y neuro-patología (33). Primero, plantean mejorar la capacidad de 
resolución de las neuroimágenes, pues las actuales son capaces de detectar infartos macroscópicos de más de $3 \mathrm{~mm}$, pero no logran detectar infartos microscópicos y enfermedad de vasos pequeños (como arterioloesclerosis). Segundo; basado en estudios de neuro-imágenes y de anatomía patológica, algunas patologías vasculares pueden representar procesos vasculares o degenerativos; pues la degeneración de sustancia blanca, medida en secuencia FLAIR y por difusión; así como los micro-sangrados están asociados tanto a TCV como a EA (48-50); además los estudios de anatomía patológica han demostrado que la degeneración de sustancia blanca y microsangrados están relacionados a lipohialinosis $(51,52)$. A su vez, la degeneración de sustancia blanca está relacionado a patología de EA y microsangrados a angiopatía amiloide cerebral (33). Por otro lado, las alteraciones del volumen de hipocampo pueden estar relacionadas a patología tipo EA o vascular (53), de tal manera que la atrofia del hipocampo puede ser en respuesta a un proceso degenerativo o vascular (54).

A la fecha, no existen criterios clínico-patológicos validados y aceptados para el diagnóstico de DMx. Peor aún, el diagnóstico de demencia vascular/trastorno cognitivo vascular (DV/TCV) sigue siendo materia de discusión debido a la heterogeneidad del fenotipo clínico-neuropsicológico producido por diversos tipos de IVC. Por lo tanto, los criterios diagnósticos de DMx sugieren emplear las recomendaciones para el diagnóstico de EA asociado a cambios morfológicos causados por cualquier tipo de IVC (55).

\section{CONCLUSIONES}

Con el paso de los años, lo que hoy conocemos como demencia ha sufrido diversas denominaciones, hasta llegar al actual enfoque clínico-biológico. Los diversos tipos de demencia se explican por alteraciones en ciertas proteínas, que determinan fenotipos clínicos característicos, capaces de ser identificados con la aplicación de los criterios diagnósticos descritos en esta revisión, la mayoría de ellos, actualizados a la fecha. Esta diferenciación diagnóstica incrementaría las posibilidades de tratamiento y mejorar el pronóstico de los pacientes afectados por estos trastornos.

\section{Correspondencia:}

Nilton Custodio

Instituto Peruano de Neurociencias

Bartolomé Herrera 161, Lince, Lima, Perú.

Telf/fax: 51-1-2653834

Correo electrónico: ncustodio@ipn.pe
Conflicto de intereses: Ninguno

\section{REFERENCIAS BIBLIOGRAFICAS}

1. Eramudugolla R, Mortby ME, Sachdev P, Meslin C, Kumar R, Anstey KJ. Evaluation of a research diagnostic algorithm for DSM-5 neurocognitive disorders in a population -based cohort of older adults. Alzheimer's Research \& Therapy. 2017; 9:15. DOI: https://doi.org/10.1186/s13195-017-0246-x

2. Taragano FE, Allegri R, Lyketsos K. Mild behavioral impairment: a prodromal stage of dementia. Dement Neuropsychol. 2008; 2(4): 256-260.

3. Chiu P-Y, Tsai C-T, Chen P-K, Chen W-J, Lai T-J. Neuropsychiatric symptoms in Parkinson's disease dementia are more similar to Alzheimer's disease than dementia with Lewy bodies: A Case-Control Study. PLoS ONE. 2016; 11(4): e0153989. doi: https://doi.org/10.1371/journal.pone.0153989

4. Dubois B, Hampel H, Feldman HH, Scheltens P, Aisen P, Andrieu S, et al. Pre-clinical Alzheimer's disease: definition, natural history and diagnostic criteria. Alzheimer's Dement. 2016; 12: 292-323.

5. Custodio NS, Wheelock A, Thumala D, Slachevsky A. Dementia in latin america: epidemiological evidence and implications for public policy. Front Aging Neurosci. 2017; 9: 221. doi: 10.3389/ fnagi.2017.00221

6. Parra M, Baez S, Allegri R, Nitrini R, Lopera F, Slachevsky A, et al. Dementia in Latin America: Assessing the present and envisioning the future. Neurology. 2017; 2018; 90:1-10. doi: 10.1212/ WNL.0000000000004897

7. Jack C Jr, Bennet DA, Blennow K, Carrillo MC, Dunn B, Budd Haeberlein S, et al. NIA-AA Research framework: Toward a biological definition of Alzheimer's disease. Alzheimer's Dement. 2018; 14: 535-562.

8. Nitrini, R. Evolución histórica del concepto de demencia y las principales enfermedades relacionadas. En: Brucky S, Magaldi R, Morillo L, Carvalho I, Perroco T, Machado C, et al. Demencias, enfoque multidisciplinario. Sao Paulo, Brasil: Editorial Atheneu; 2011.

9. Slachevsky A, Oyarzo F. Las demencias: historia, clasificación y aproximación clínica. En: Labos E, Slachevsky A, Fuentes P, Manes F. Tratado de Neuropsicología Clínica. Buenos Aires, Argentina: Librería Akadia editorial; 2008.

10. Slachevsky A. Las demencias: historia, concepto, clasificación y dificultades diagnósticas. En: Guajardo G. Salud mental y personas mayores: Reflexiones teórico-conceptuales para la investigación social de las demencias. Santiago de Chile, Chile: FLACSOChile; 2016. 
11. Berríos GE. Dementia: Historical Overview. En: Burns A, O’Bryen J, Ame D, eds. Dementia. London: Hodder Arnold; 2005.

12. Caixeta L, Lima JN, Marques AC, Nóbrega M. The development of the dementia concept in 19th century. Arq Neuropsiquiatr. 2014; 72(7): 564-567.

13. García-Albea E. Areteo de Capadocia (siglo II d.C.) y las primeras descripciones neurológicas. Rev Neurol. 2009; 48: 322-327.

14. George DR, Whitehouse PJ, Ballenger J. The evolving classification of dementia: placing the DSM-IV in a meaningful historical and cultural context and pondering the future of "Alzheimer's". Cult Med Psychiatry. 2011; 35: 417-435.

15. Alvarez JP. Jean-Étienne Dominique Esquirol, aliéniste. Rev Med Clin Condes. 2012; 23(5): 644645.

16. Villasante O. La introducción del concepto de "parálisis general progresiva" en la psiquiatría decimonónica española. Aesclepio. 2000; 52 (1): 5372.

17. Caponi S. Para una genealogía de la anormalidad: la teoría de la degeneración de Morel. Scientae studia Sao Paulo. 2009; 7(3): 425-445.

18. Maurer K, Volk S, Gerbaldo H. Auguste D and Alzheimer's disease. Lancet. 1997; 349: 1546-1549.

19. Kertesz A, Kalvach P. Arnold Pick and German neuropsychiatry in Prague. Arch Neurol. 1996; 53: 935-938.

20. McKhann G, Drachman D, Folstein M, Katzman R, Price D, Stadlan EM, et al. Clinical diagnosis of Alzheimer's disease: report of the NINCDS-ADRDA Work Group under the auspices of Department of Health and Human Services Task Force on Alzheimer's Disease. Neurology. 1984; 34: 939-44.

21. Asociación Americana de Psiquiatría. DSM-IV Manual diagnóstico y estadístico de los trastornos mentales, Cuarta edición . Washington: British Library; 1994.

22. Organización Mundial de la Salud. Clasificación Internacional de Enfermedades, décima edición (CIE10). Geneva: Organización Mundial de la Salud; 1992.

23. Asociación Americana de Psiquiatría. DSM-5 Manual diagnóstico y estadístico de los trastornos mentales, quinta edición. Madrid: Editorial Panamericana; 2014.

24. Petersen RC, Smith GE, Waring SC, Ivnik RJ, Tangalos EG, Kokmen E, et al. Mild cognitive impairment: clinical characterization and outcome. Arch Neurol. 1999; 56: 303-308.

25. López-Alvarez J, Agüera-Ortiz LF. Nuevos criterios diagnósticos de la demencia y la enfermedad de Alzheimer: una visión desde la psicogeriatría. Psicogeriatría. 2015; 5(1): 3-14.

26. Dubois B, Feldman HH, Jacova C, DeKosky ST,
Barberger-Gateau P, Cummings J, et al. Research criteria for the diagnosis of Alzheimer's disease: revising the NINCDS-ADRDA criteria. Lancet Neurol. 2007; 6: 734-746.

27. McKhann GM, Knopman DS, Chertkow H, Hyman BT, Jack Jr CR, Kawas CH, et al. The diagnosis of dementia due to Alzheimer's disease: recommendations from the National Institute on Aging-Alzheimer's Association work groups on diagnostic guidelines for Alzheimer's disease. Alzheimer's Dement. 2011; 7: 263-269.

28. Custodio N, Herrera-Pérez E, Lira D, Montesinos R, Linares J, Bendezú L. Deterioro cognitivo leve: Dónde termina el envejecimiento normal y empieza la demencia? An Fac Med. 2012; 73(4): 321-330.

29. Petersen R, Stevens J, Ganguli M, Tangalos E, Cummings J, DeKosky S. Practice parameter: early detection of dementia: Mild cognitive impairment (an evidence-based review). Report of the Quality Standards Subcommittee of the American Academy of Neurology. Neurology. 2001; 56: 1133-1142.

30. Sperling R, Aisen P, Beckett L, Bennett D, Craft S, Fagan A, et al. Towards defining the preclinical stages of Alzheimer's disease: recommendations from the National Institute on Aging- Alzheimer's Association work groups on diagnostic guidelines for Alzheimer's disease. Alzheimer's \& Dementia. 2011; 7: 280-292.

31. Hannon B, Daneman M. Age-related changes in reading comprehension: an individual-differences perspective. Exp Aging Res. 2009; 35: 432-456.

32. Roman GC, Tatemichi TK, Erkinjuntti T, Cummings JL, Masdeu JC, Garcia JH, et al. Vascular dementia: diagnostic criteria for research studies. Report of the NINDS-AIREN International Workshop. Neurology. 1993; 43: 250-260.

33. Gorelick PB, Scuteri A, Black SE, De Carli C, Greenberg SM, Iadecola $C$, et al. Vascular contributions to cognitive impairment and dementia: a statement for healthcare professionals from the American heart association/American stroke association. Stroke. 2011; 42: 2672-2713.

34. Custodio N, Montesinos R, Alva-Diaz C, MejíaRojas K, Becerra-Becerra Y, Lira D. Nuevos términos clínicos, prevención y tratamiento del trastorno cognitivo vascular: revisión de la literatura basada en la evidencia. Rev Neuropsiquiatr. 2016; 79(3): 152165.

35. Mimenza AJ, Cantú CG, Román G, Gareri P, Aguilar SG, Ruiz Sandoval JL, et al. Latin American Delphi Consensus on Vascular Cognitive Impairment: Definitions, clinical features, pathophysiology, prevention and treatment. J Neurol Neurosci. 2017; 8(5): 224.

36. Gorelick PB, Counts SE, Nyenhuis D. Vascular cognitive impairment and dementia. Biochim 
Biophys Acta. 2016; 1862(5): 860-868.

37. Rascovsky K, Hodges J, Knopman D, Mendez M, Kramer J, Neuhaus J, et al. Sensitivity of revised diagnostic criteria for the behavioural variant of frontotemporal dementia. Brain. 2011; 134: 24562477.

38. Gorno-Tempini M, Hillis A, Weintraub S, Kertesz A, Mendez M, Cappa S, et al. Clasification of primary progressive and its variants. Neurology. 2011; 76: 1006-1014.

39. Neary D, Snowden JS, Gustafson L, Passant U, Stuss D, Black S, et al. Frontotemporal lobar degeneration: a consensus on clinical diagnostic criteria. Neurology. 1998; 51: 1546-1554.

40. Piguet O, Hornberger M, Mioshi E, Hodges J. Behavioural-variant frontotemporal dementia: diagnosis, clinical staging and management. Lancet Neurol. 2011; 10: 162-72.

41. Reul S, Lohmann H, Wiendl H, Duning T, Johnen A. Can cognitive assessment really discriminate early stages of Alzheimer's and behavioural variant frontotemporal dementia at initial clinical presentation? Alzheimer's Research \& Therapy. 2017; 9: 61. doi: 10.1186/s13195-017-0287-1

42. Miki T, Yokota O, Ishizu H, Kuroda S, Oshima E, Terada S, et al. Behavioral variant of frontotemporal dementia: fundamental clinical issues associated with prediction of pathological bases. Neuropathology. 2016; 36: 388-404.

43. McKeith IG, Boeve BF, Dickson DW, Glenda H, Taylor J, Weintraub D, et al. Diagnosis and management of dementia with Lewy bodies: Fourth consensus report of the DLB Consortium. Neurology. 2017; 89: 1-13.

44. McKeith IG, Dickson DW, Lowe J, Emre M, O’Brien JT, Feldman H, et al. Diagnosis and management of dementia with Lewy bodies: Third report of the DLB Consortium. Neurology. 2005; 65: 1863-1872.

45. Custodio N, Montesinos R, Lira D, Herrera-Pérez E, Bardales Y, Valeriano-Lorenzo L. Mixed dementia: A review of the evidence. Dement Neuropsychol. 2017; 11(4): 364-370.

46. Mirra SS, Heyman A, McKeel D, Sumi SM, Crain BJ, Brownlee LM, et al. The Consortium to Establish a Registry for Alzheimer's Disease (CERAD). Part
II. Standardization of the neuropathologic assessment of Alzheimer's disease. Neurology. 1991; 41: 47986.

47. Chui HC, Victoroff JI, Margolin D, Jagust W, Shankle R, Katzman R. Criteria for the diagnosis of ischemic vascular dementia proposed by the State of California Alzheimer Disease Diagnostic and Treatment Centers. Neurology. 1992; 42(3):473-480.

48. Kirsch W, McAuley G, Holshouser B, Petersen F, Ayaz M, Vinters HV, et al. Serial susceptibility weighted MRI measures brain iron and microbleeds in dementia. J Alzheimers Dis. 2009; 17: 599-609.

49. Cordonnier C, van der Flier WM, Sluimer JD, Leys D, Barkhof F, Scheltens P. Prevalence and severity of microbleeds in a memory clinic setting. Neurology. 2006; 66: 1356-1360.

50. Lee DY, Fletcher E, Martinez O, Ortega M, Zozulya $\mathrm{N}$, Kim J, et al. Regional pattern of White matter microstructural changes in normal aging, $\mathrm{MCI}$, and AD. Neurology. 2009; 73:1722-1728.

51. Fazekas F, Kleinert R, Roob G, Kleinert G, Kapeller $\mathrm{P}$, Schmidt R, et al. Histopathologic analysis of foci of signal loss on gradient-echo T2*-weighted MR images in patients with spontaneous intracerebral hemorrhage: evidence of microangiopathy-related microbleeds. AJNR Am J Neuroradiol. 1999; 20: 637-642.

52. Tanaka A, Ueno Y, Nakayama Y, Takano K, Takebayashi S. Small chronic hemorrhages and ischemic lesions in association with spontaneous intracerebral hematomas. Stroke. 1999; 30: 16371642.

53. Jagust WJ, Zheng L, Harvey DJ, Mack WJ, Vinters HV, Weiner MW, et al. Neuropathological basis of magnetic resonance images in aging and dementia. Ann Neurol. 2008; 63: 72-80.

54. Zarow C, Sitzer TE, Chui HC. Understanding hippocampal sclerosis in the elderly: epidemiology, characterization, and diagnostic issues. Curr Neurol Neurosci Rep. 2008; 8: 363-370.

55. Jellinger KA, Atems J. Neuropathological evaluation of mixed dementia. J Neurol Sci. 2007; 257: 80-87.

Recibido: 17/09/2018

Aceptado: 19/11/2018 